\title{
Orientations and Crystallinities of Drawn Fibers Using Two Beam Interferometry
}

\author{
A. M. Ali ${ }^{1,2}$ \\ ${ }^{1}$ Physics Department, Faculty of Science, Umm Al-Qura University, KSA \\ ${ }^{2}$ Physics Department, Faculty of Science, Mansoura University, Egypt
}

\begin{abstract}
T $\mathrm{N}$ this work, the Pluta interference microscope was used to study the effect of drawing process on the optical, structural, and mechanical properties of polyethylene terephthalate (PET) fibers. The orientational behavior of drawn PET fibers to different draw ratios was investigated using the calculated values of the refractive indices and the double refraction. Changing the draw ratio resulted in changes in optical parameters, which were used to determine the mechanical factors of orientation. Different orientation functions, such as $f_{2}(\theta), f_{4}(\theta), f_{6}(\theta), f_{a}, f_{c}$, and $f_{\mathrm{av}}$, were calculated. Molecular reorientation during the cold drawing process can be considered the main reason for the major variations in the properties of PET fibers. It can be concluded that double refraction related to the total overall orientation of the crystalline and amorphous phases. The different crystallinities parameters were measured. Microinterferograms were utilized to illustrate these variations.
\end{abstract}

Keywords: Orientation, Crystallinity, Drawing and PET fibers.

\section{Introduction}

Polyethylene terephthalate (PET), a semi crystalline polymer, can be used in a wide range of commercial applications due to its desirable physical and chemical properties, such as clearness, light weight, high strength, stiffness, high chemical resistance, elastic nature, and low price [1-3]. A drawing process should be applied to make PET fibers acceptable for textile and other industrial applications. The polymer tensile deformations are always accompanied by advance alignment of chains along the drawing direction. The amorphous and crystalline molecular orientation is related to the induced strain in the sample. There are two different models that explain the orientation as a function of draw ratio the pseudo- affine and affine models. The application of one these model depends on the drawing conditions [4]. Many authors studied the effect of drawing process on the mechanical and structural behavior of PET [5-9].

To enhance the behavior of the final polymeric products, the orientation amount and crystallinity degree should be completely characterized. Different experimental methods, such as wideangle X-ray scattering, X-ray scattering, and density measurement, can be used to monitor these properties (Kolb et al., 2000). Several methods, such as molten fiber stretching, rapid cooling, and hot or cold drawing process, can be utilized to produce orientation in the polymers. Fiber properties can be changed by the drawing process. This change is due to the molecular arrangement, which is vital in characterizing the orientation, crystallinity, and other structural properties. When polymers, such as PET, are mechanically drawn above or close to their glass transition temperatures, an induced strain crystallinity can be formed.

Birefringence is a physical indicator that correlates the mechanical and optical properties of fiber. Measuring the birefringence and correlating it to other physical properties shed light on the characteristics of a bulk polymer. An external force is applied to the fiber during the cold drawing process so that the polymer chains become highly oriented along the drawing direction $[11,12]$. Birefringence measurement can be used as a significant indicator to characterize the amount of orientation and anisotropy. The birefringence of polymeric fibers has been extensively investigated because it is related to the polarizability of the molecules and the orientation function of these fibers. Birefringence and refractive indices provide an orientation measure, which is an average of the amorphous 
and crystalline regions [13,14]. Major changes in the fiber structure can be achieved as a result of the drawing process. The amount of orientation in the fibers increases with the increase in stress applied during the drawing process.

PET can be considered a crystallizable polymer due to the symmetry and regularity of its geometrical and chemical structures. The crystallinity degree and morphology exert considerable effects on different polymer properties. One of the most important factors affecting crystallization is stress. The application of stress on PET fibers leads to the formation of stress-induced crystallization, which can be measured using different techniques, such as density measurements and X-ray diffraction [15].

The present work aims to illustrate the effects of the cold drawing process on the optical, mechanical, electrical, and structural properties of PET fibers. All of the desired properties can be characterized using a drawing device coupled to a two-beam interference microscope.

\section{Theoretical consideration}

Refractive indices and double refraction measurement

The fiber refractive indices can be measured using the following equation [14]:

$$
n=n_{l}+\frac{F \lambda}{b A}
$$

Where is the refractive index of immersed liquid, is the area enclosed under the fringe shift, is the light wavelength, $A$ is the cross-sectional area of the filament under study, and $b$ is the spacing between two successive fringes.

Birefringence can be evaluated using the following equation [14] with the aid of the obtained refractive indices.

$$
\Delta n=n \mathbf{I}-n^{\perp}
$$

Determination of different orientation functions

The degree of molecular orientation of polymers mostly depends on the Herman's orientation factor that relates between the oriented birefringence $(\Delta n)$ and the maximum intrinsic birefringence $\left(\Delta \mathrm{n}_{\max }=0.24\right)$ [16], as expressed in the following equation $[17,18]$.

$$
f(\theta)=\frac{\Delta n}{\Delta n_{\max }}
$$

Herman's orientation factor represents $f(\theta)$ as the orientation function in terms of the Fourier series, as shown in the following equation [19]:

$$
\left.f(\theta)=\sum_{n=\mathrm{o}}^{\infty} \mathrm{r}\left(n+\frac{1}{2}\right)\right)<f_{n}>f_{n}(\theta)
$$

Given that the odd terms of this series all are zero, the first three even terms are expressed as follows:

$$
\begin{aligned}
& f_{4}(\theta)=\frac{35 \cos ^{4}(\theta)-3 \cos ^{2}(\theta)+3}{8} \\
& f_{2}(\theta)=\frac{3 \cos ^{2}(\theta)-1}{2} \\
& f_{6}(\theta)=\frac{231 \cos ^{6}(\theta)-15 \cos ^{4}(\theta)+150 \cos ^{2}(\theta)-5}{6}(4)
\end{aligned}
$$

where is the angle stuck between the oriented chain and the fiber axis (intial inclinations). The total orientational angle can be measured using the following equation:

$$
\theta=\sin ^{-1}[-(1-f(\theta))]^{\frac{1}{2}}
$$

With the drawing process applied to the fiber, its segments will be constrained by a certain angle (Ø). The constraining angle (the final inclinations) can be evaluted with the aid of the following expression:

$$
\tan (\emptyset)=D R^{\frac{-3}{2}} \tan (\theta)
$$

Orientation means phase ordering. Crystalline and amorphous materials can be observed in the oriented or unoriented phase. $f_{a v}$ is the average orientation value, which can be measured using the following equation [20]:

$$
f_{a v}=\frac{2 \Delta n}{\left(\Delta n_{c}^{o}+\Delta n_{a}^{o}\right)}
$$

where $\Delta n_{C}^{O}$ is the interinsic birefringence of the crystailine region, and is the interinsic birefringence of the amorphous region. The interinsic birefringence is birefringence obtained at complete orientation of the materialwhen $\operatorname{Sin}^{2} \theta=0$. For PET fibers [21] ,

$$
\Delta n_{c}^{o}=0.22, \Delta n_{a}^{o}=0.275 \text {. }
$$

The orientation function of the crystalline phase $\left(f_{c}\right)$ can be characterized by using the following equation [22] and determining the draw ratio (DR):

$$
f_{C}=\frac{\left(D R^{3}-1\right)}{\left(D R^{2}+2\right)}
$$

The following expression can be used to measure the orientation function of the amorphous phase. 


$$
f_{a}=f(\theta)-f_{c}
$$

The individual segment orientation using integral functions is used to evaluate the average molecular orientation of the segments.

$<\cos ^{2}(\emptyset)>=\int_{0}^{\frac{\pi}{2}} \cos ^{2}(\varphi) \sin (\theta) d \theta$

Where $\left\langle\operatorname{Cos}^{2}(\emptyset)\right\rangle$ is the average cosine square of the included angle between molecular segment and the drawing direction. Herman's orientation function $\left\langle P_{2}\right\rangle$ is given by the following equation:

$\left\langle P_{2}\right\rangle=\frac{3\left\langle\cos ^{2}(\emptyset)\right\rangle-1}{2}$

Since there is no single unit but there is an aggregate unit so,

$<\cos ^{2}(\varnothing)>=\int_{0}^{\frac{\pi}{2}} \frac{D^{3} \sin \theta}{D^{2}+\tan ^{2} \theta} d \theta$

Solving the above equation:

$<P_{2}>=\frac{3}{2}\left[\frac{D^{3}}{D^{2}-1}-\frac{D^{3}}{\sqrt{\left(D^{3}-1\right)^{3}}} \operatorname{Arctan}\left(\sqrt{D^{2}-1}\right)\right]-\frac{1}{2}$

As clear, the average mechanical orientation depend only on the draw ratio. According to the aggregate model, mechanical anisotropy due to the application of a low-strain drawing process is correlated with the mechanical molecular orientation functions. If the network is considered random links, then these links are identical and consisted of freely jointed chains (pseudo- affine model).

The molecular polarizability of polymer

$P_{m}$ "the molecular Polarizability"can be measured using the following equation [23].

$p_{m}=\frac{3}{4 \pi}\left(\frac{n_{a}^{2}-1}{n_{a}^{2}+2}\right)$

The mean refractive index value can be calculated using the following equation.

$$
n_{a}=\frac{n_{\|}+2 n_{\perp}}{3}
$$

\section{Determination of dielectric constant}

When measuring the radically dielectric constant, the refractive index values $(n)$ will be used as the parallel refractive index. When measuring the axially dielectric constant, the perpendicular refractive index values will be used.

$$
D E_{\cdots}=n^{2}
$$

\section{Determination of crystallinity}

The mechanical drawing process considerably influences a material. This effect can be examined by measuring density using the calculated refractive indices according to the Devrise equation $[22,24]$.

$$
\rho=4.047 \frac{\left(n^{2}-1\right)}{\left(n^{2}+2\right)}
$$

The volume fraction crystallinity of the treated PET fibers can be calculated using the measured values of the density using the following equation(Jackson, 1975):

$$
x_{v}=\frac{\rho-\rho_{a}}{\rho_{c}-\rho_{a}}
$$

Where $\left(\rho_{c}=1.457 \frac{\mathrm{g}}{\mathrm{cm} 3}\right)$ and $\left(\rho_{a}=1.336 \frac{\mathrm{g}}{\mathrm{cm} 3}\right)$.

The weight fraction crystallinity can be measured using the equation:

$$
x_{w}=\frac{\rho-\rho_{a}}{\rho_{c}-\rho_{a}} \frac{\rho_{c}}{\rho_{a}}
$$

The volume fraction of amorphous material can be evaluated using the given equation:

$$
1-x_{v}={ }_{1}-\frac{\rho-\rho_{a}}{\rho_{c}-\rho_{a}}
$$

\section{Experimental setup}

A two-beam interference microscope [25] is an accurate device widely used in the field of fiber research. The obtained images are used to measure the different optical and structural properties of the fiber. A mechanical device is designed for analysis of the different properties of drawn fibers [26].The designed cold drawing device attached to the two-beam interference microscope was used to understand the behavior of drawn fibers, such as optical and structural properties. The ends of the fiber between the two clamps were fixed using an adhesive material. The fiber was immersed in a liquid with a refractive index $n_{l}$. Afterward, the drawing device was transferred to the microscope stage. CCD camera was used to record the fiber image during the drawing process. The obtained microinterferograms were used with the previously presented equations to calculate the required properties of fibers at different draw ratios (D). The samples were drawn until breaking down at draw ratio $(\mathrm{D}=2)$.

\section{Results and Discussions}

A filament of PET fiber between the clamps of the device was fixed and immersed in a liquid with a refractive index of 1.604. The temperature $(T)$ was $30^{\circ} \mathrm{C}$. The wavelength used was $546.1 \mathrm{~nm}$. Figure 2 shows the obtained microinterferograms of the duplicated images of PET fiber at different 


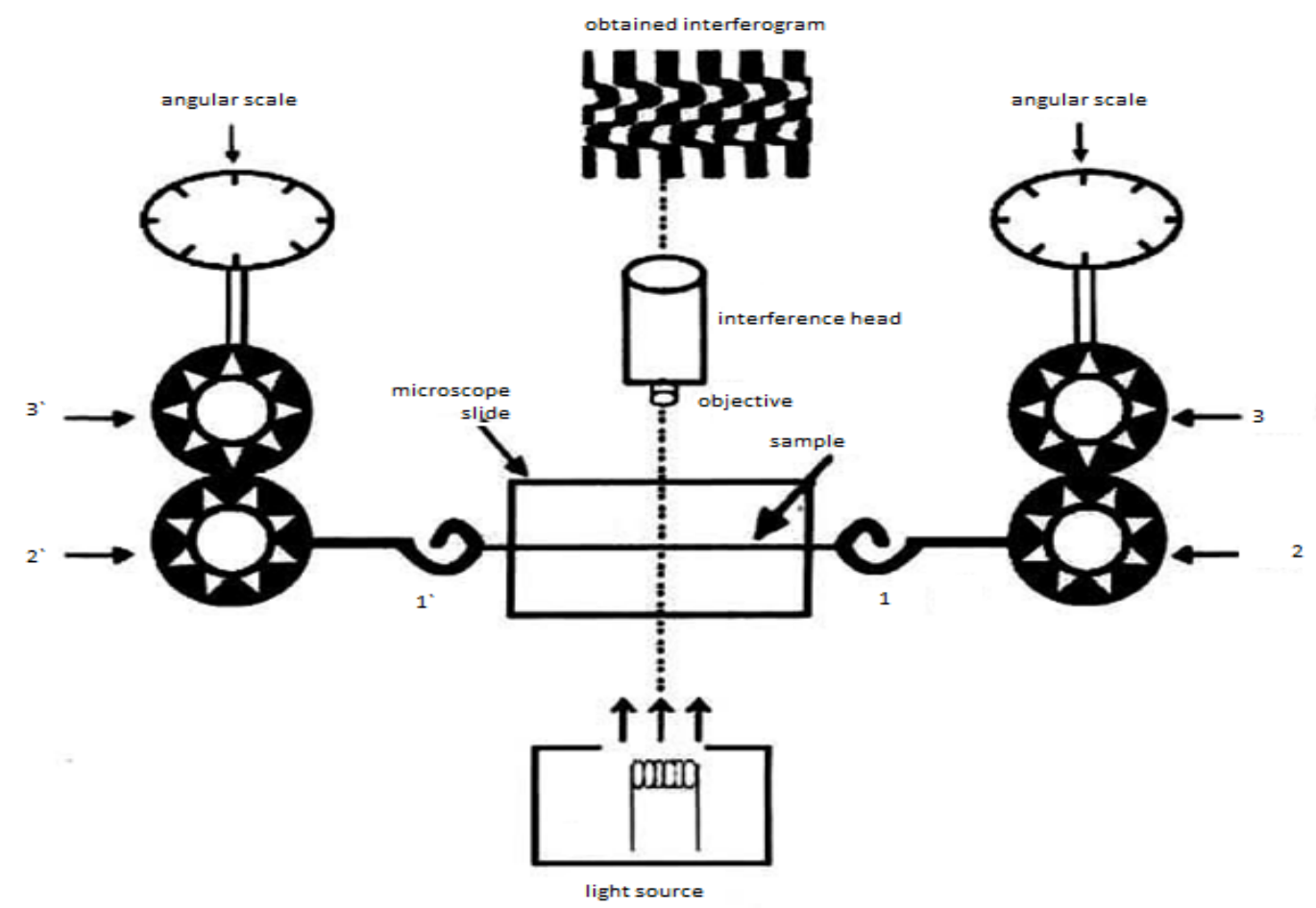

Fig. 1. A schematic diagram of the rotator device attached with the Pluta microscope where: 1, 1: Two clamps, 2, 2`:Two gears connected to the clamps. 3, 3`: Two intermediate gears, Two angular scales, Microscope slide, Sample under study.

Interference head. Objective., Microinterferogram., Iluminating source [26].

draw ratio ( D). The refractive indices $n^{\|}$and $n^{\wedge}$ at each $\mathrm{D}$ were measured using the images shown in Fig. 2 and Equation (1).

Figure 3 presents the calculated refractive indices at different DRs. The parallel refractive index increases with the increase in $\mathrm{D}$, as shown in Fig. 3. Thus, the fiber chain becomes highly oriented in the parallel direction. The axial packing improves during the drawing process. However, the refractive index values for the perpendicular direction decrease with the increase in DR.

The double refraction can be calculated using Equation (2). Birefringence increases with the increase in D, as shown in Fig. 4. Notably, the fibers constituting molecules are more oriented in the parallel direction than in the perpendicular direction. This finding indicates that the axial packing largely improves, and the radial packing slightly decreases, thereby resulting in an increase in the resultant birefringence.

The orientation function $f(\theta)$ was measured using the calculated birefringence with the aid of Equation (3). Table 1 provides a list of the calculated orientation function values with the D. With the increased $\mathrm{D}$, the computed orientation function increases. The different optical orientation functions, namely, $f_{2}(\theta), f_{4}(\theta)$, and $f_{6}(\theta)$, were calculated at different Ds. The obtained results are tabulated in Table 1. With the increased $\mathrm{D}$, all of the computed optical orientation functions increase. These calculated data can be used as a useful prediction key for the amount of optical and mechanical anisotropies in the samples under study. The orientation function increases linearly with birefringence.Therefore, the molecular arrangements in the amorphous and crystalline regions are a result of the orientation that occurred.

The crystalline and amorphous orientation functions $\left(f_{c}\right.$ and $\left.f_{a}\right)$ were measured using Equations (8) and (9), and results are tabulated in Table 1. With the increased $\mathrm{D}$, the obtained $f$ and $f_{c}$ values increase, as clearly shown in Table (1). Evidently, $f_{c}$ exhibits considerable changes at all Ds, whereas $f_{a}$ increases slowly with increased 


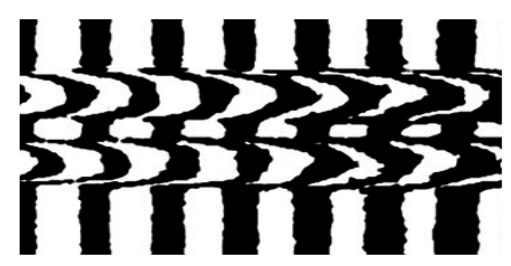

(a)

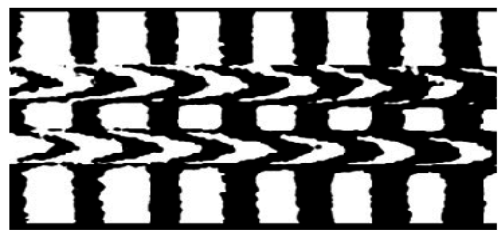

(c)

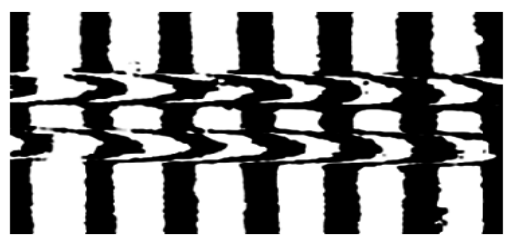

(e)

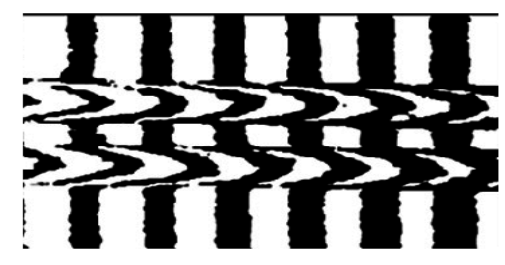

(b)

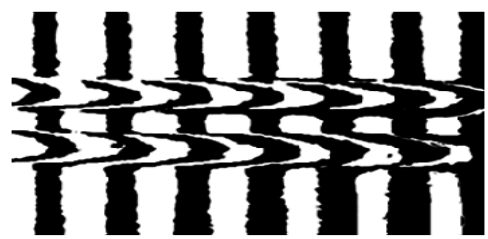

(d)

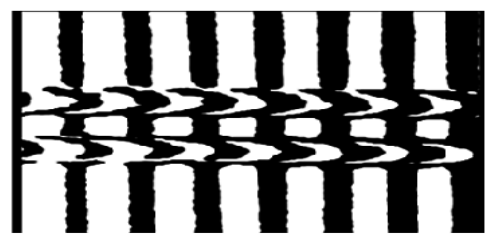

(f)

Fig. 2. The obtained duplicated images microinterferograms of PET fiber at different draw ratio.

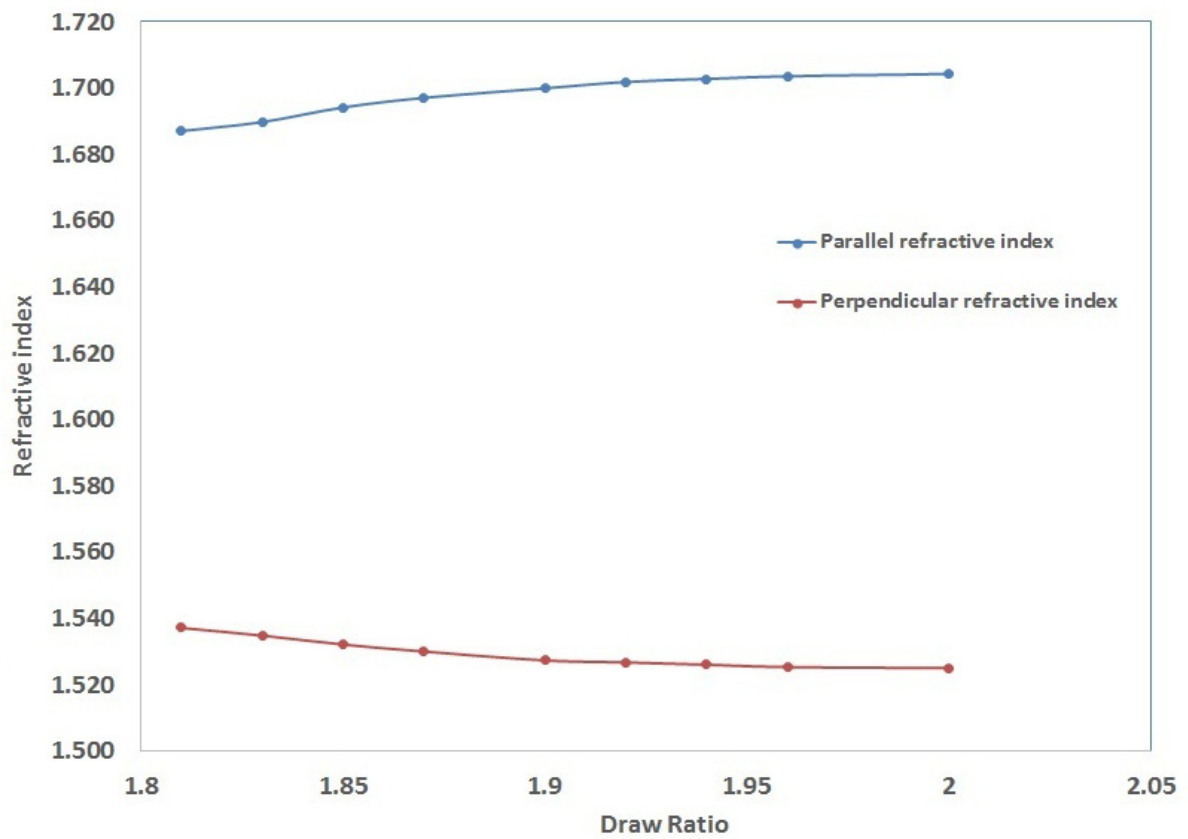

Fig. 3.The variation of the refractive indices $\mathbf{n}^{\|}$and $\mathbf{n}$ with draw ratio. 


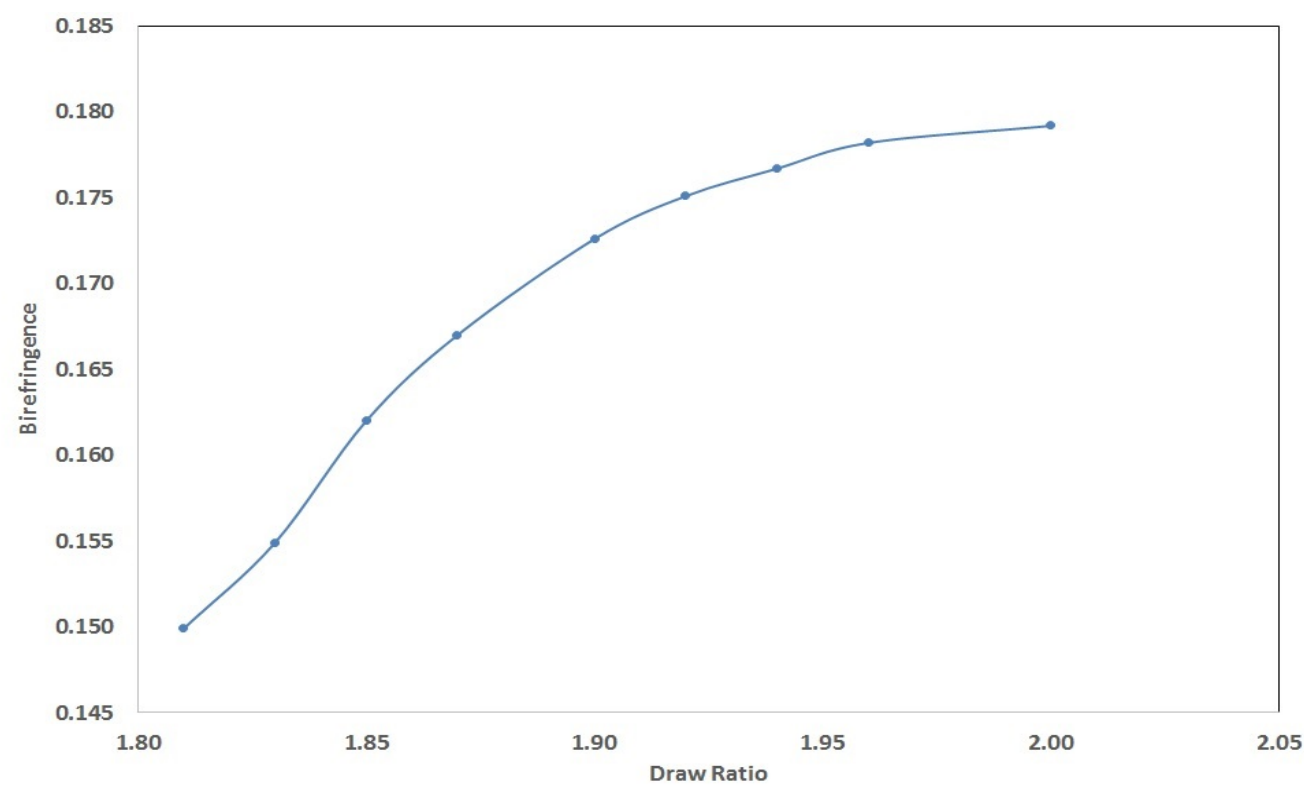

Fig. 4. The variation of birefringence with the draw ratio.

TABLE 1. The different calculated orientations for mechanically drawn PET fibers.

\begin{tabular}{cccccccc}
\hline Draw ratio & $\mathbf{f}(\mathbf{q})$ & $\mathbf{f}(\mathbf{q})$ & $\mathbf{f}_{\mathbf{4}}(\mathbf{q})$ & $\mathbf{f}_{\mathbf{6}}(\mathbf{q})$ & $\mathbf{f}_{\mathbf{a v}}$ & $\mathbf{f}_{\mathbf{c}}$ & $\mathbf{f}_{\mathbf{a}}$ \\
\hline 1.81 & 0.6245 & 0.6249 & 0.0233 & 27.12 & 0.6057 & 0.6217 & 0.0029 \\
1.83 & 0.6454 & 0.6432 & 0.0582 & 28.09 & 0.6259 & 0.6309 & 0.0145 \\
1.85 & 0.6750 & 0.6748 & 0.1217 & 29.84 & 0.6545 & 0.6399 & 0.0351 \\
1.87 & 0.6958 & 0.7015 & 0.1783 & 31.37 & 0.6747 & 0.6487 & 0.0472 \\
1.9 & 0.7192 & 0.7209 & 0.2212 & 32.52 & 0.6974 & 0.6614 & 0.0578 \\
1.92 & 0.7296 & 0.7394 & 0.2634 & 33.64 & 0.7075 & 0.6695 & 0.0601 \\
1.94 & 0.7363 & 0.7489 & 0.2858 & 34.23 & 0.7139 & 0.6775 & 0.0588 \\
1.96 & 0.7425 & 0.7641 & 0.3218 & 35.18 & 0.7199 & 0.6852 & 0.0573 \\
2 & 0.7467 & 0.7840 & 0.3707 & 36.46 & 0.7240 & 0.7000 & 0.0467 \\
\hline
\end{tabular}

DR. This finding suggests the rapid growth in crystalline orientation factor and the slow development in the amorphous orientation factor. The average orientation function $f_{a v}$ increases with the increased D, as shown in Table 1. This increase can be explained on the basis of the flexible chain behavior of the fibers. The chain segment mobility and the orientation ability increase during the drawing process.

The mechanical orientation function can be measured using Equation (11). Figure 5 illustrates the variation in mechanical orientation functions $\left.<P_{2}\right\rangle$ with the $\mathrm{D}$. The calculated data show that the mechanical orientation function ismechanically dependent. The calculated values of is comparatively small. Figures 6 (a), (b), Egypt. J. Phys. Vol. 46 (2018) and 6(c) show the variation in different optical orientation functions with birefringence, namely, (a) $f_{2}(\theta)$, (b) $f_{4}(\theta)$, and (c) $f_{6}(\theta)$. Fig. 7 presents that the different optical orientation functions calculated increase with the linear increase in the fiber birefringence.

The molecular arrangements in the crystalline and amorphous regions result in many orientations (Rossignol et al., 1989). Figures 7(a \& b) present the variations in with the draw ratio $D$. With the increased $\mathrm{D}$, the tangents of the angles decrease, as shown in Figure 6. For nondrawn samples, the chain segment is randomly oriented with an angle $\theta$. As a result of the drawing process, these chain segments are constrained at angle . 


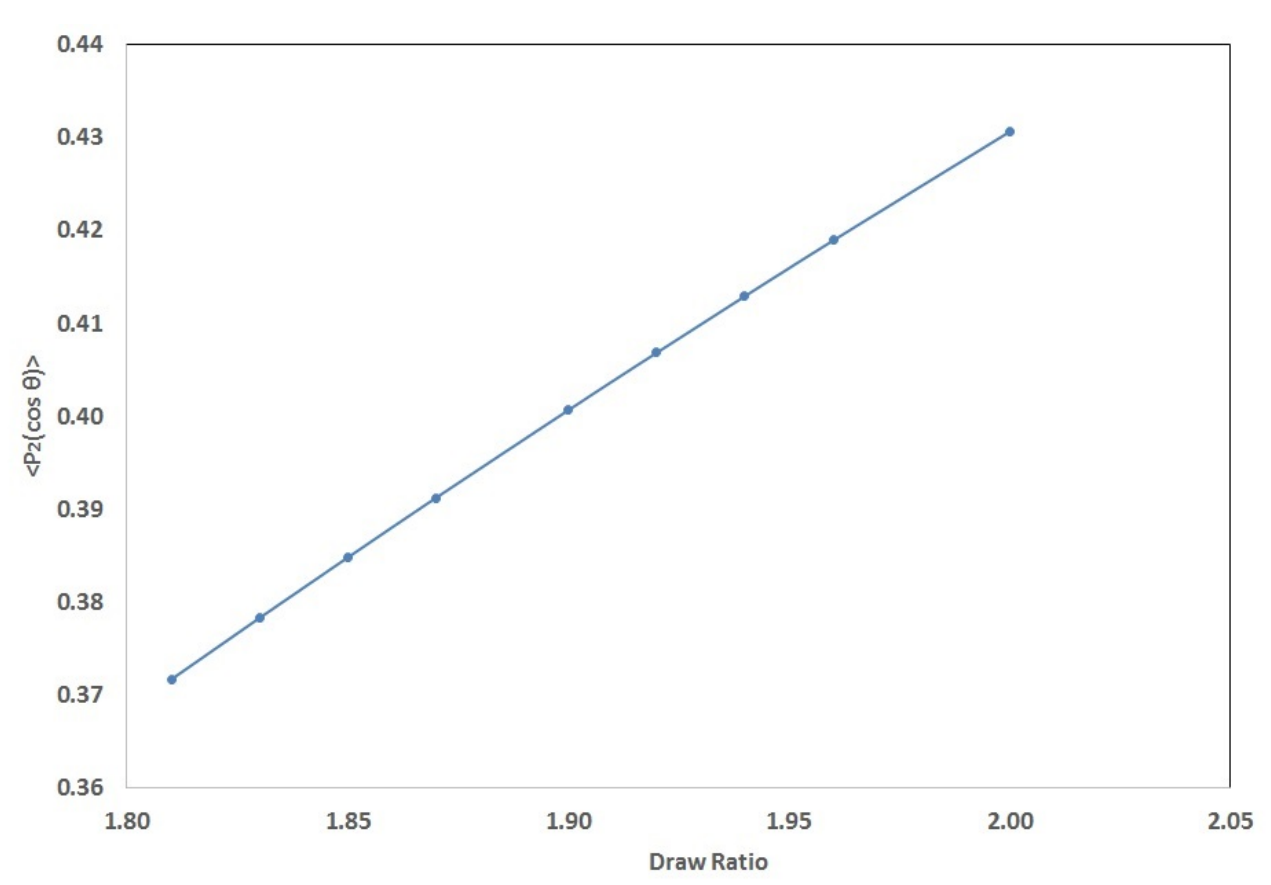

Fig. 5. The variation of mechanical orientation functions $\left\langle P_{2}\right\rangle$ with the draw ratio.

The molecular polarizability of the PET fibers was calculated using Equations (12) and (13). Figure 8 shows the variation in molecular polarizability $\left(p_{m}\right)$ with the D. Thus, with the increased $\mathrm{D}$, the molecular polarizability decreases. The previously measured optical parameters and its variation can be considered a result of the adjustment in electrical properties. The dielectric constants were measured using Equation (14). Figure 9 (a) and (b) shows the dielectric constant variations with the $\mathrm{D}$ for parallel and perpendicular directions, respectively. With the increased $\mathrm{D}$, the parallel dielectric constant increases (Fig. 9 (a), whereas the perpendicular dielectric constant decreases (Fig. 9 (b). Therefore, the drawing process affects the electrical and other physical properties of fiber.

The polymer chains become closely packed and parallel to one another in the crystalline phase. By contrast, the polymer chains become disordered in the amorphous phase. Crystallinity can be induced by the drawing and thermal treatment processes [28]. The degree of molecular crystallinity depends mainly on two common factors, namely, intrinsic and extrinsic factors. Intrinsic factors are the linear chain structure and high molecular weight of the polymer. The extrinsic factors are the draw ratio and drawing mode. In PET fibers, crystallinity can be induced by strain-induced and thermal-induced crystallizations. Stress is a vital factor affecting the crystallization process of PET. Table 2 presents the calculation results of the different types of crystallinity with the D. Notably, with the increased draw ratio, the volume and weight fraction crystallinities decrease. As a result of the cold drawing process of PET fibers, high double refraction values are obtained, which results in significant chain orientations. Consequently, these chain alignments lead to high crystallinity values. During the cold drawing process, small spherulites are formed as a result of the formation of nuclei centers (Misra and Stein, 1979).

The drawing process of PET fibers is described as a result of three different stages (Salem, 1999), (Huisman and Heuvel, 1989). In the first stage, the chains slip past one another due to the extension in the amorphous chains. In the second stage, chain uncoiling occurs because of the continuous stress application, but this stage happens occurs at a low stress level. In the third stage, strain-induced crystallization occurs at the same direction as that of the applied stress. 
A. M. ALI

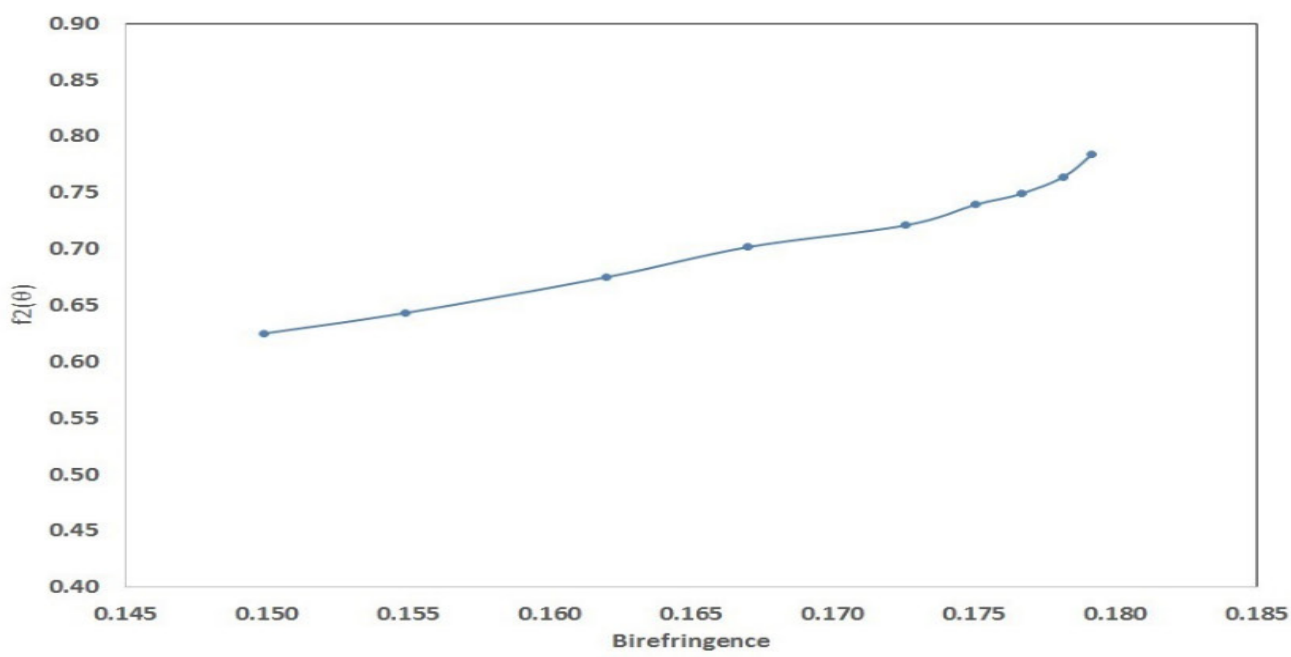

(a)

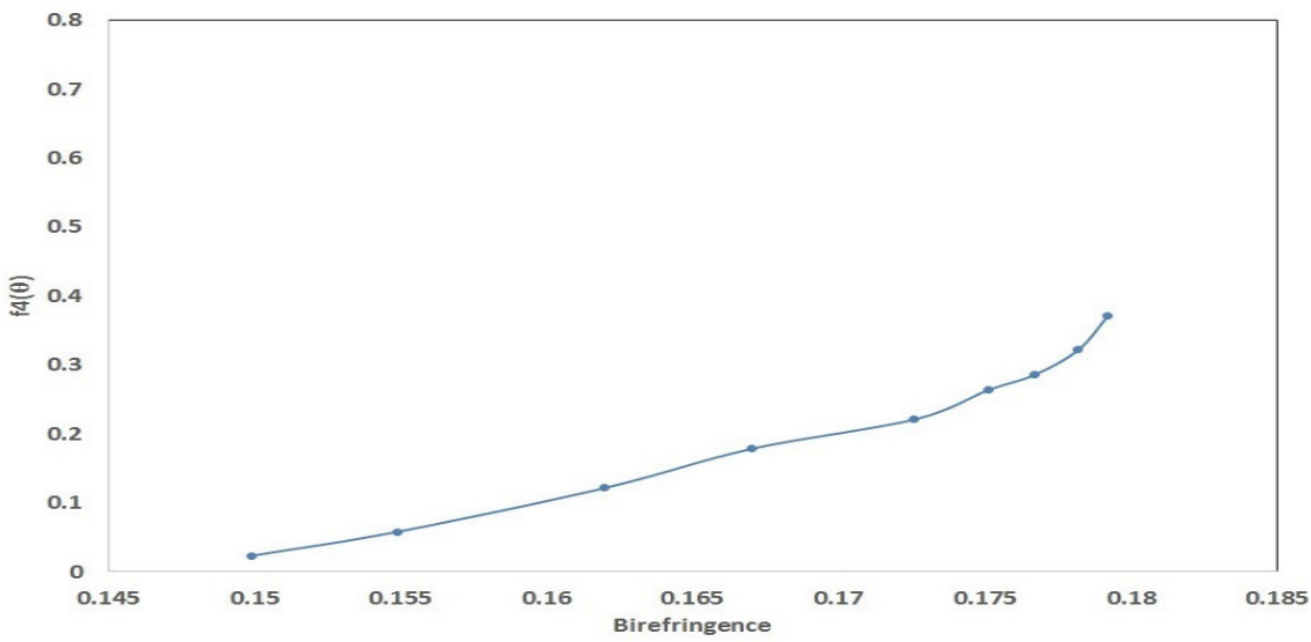

(b)

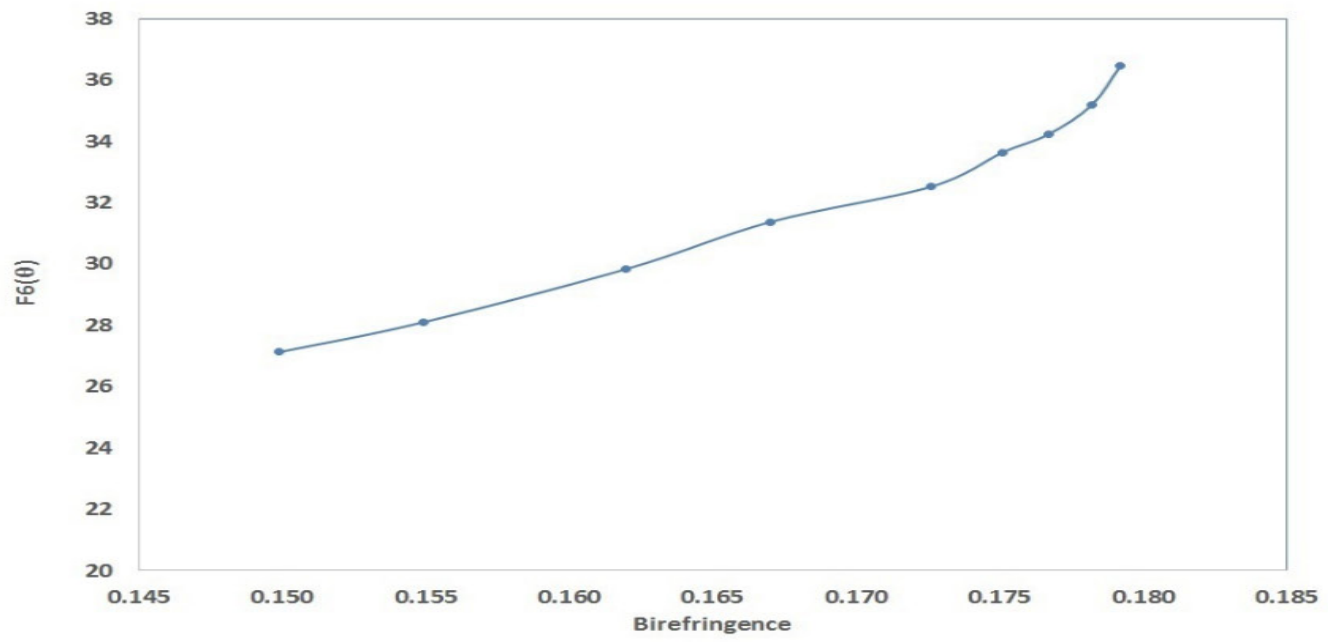

Fig. $6(a, b, c)$. The variation of the different optical orientation functions with the birefringence, a) $\left.f_{2}(\theta), b\right) f_{4}(\theta)$ and c) $f_{6}(\theta)$.

Egypt. J. Phys. Vol. 46 (2018) 

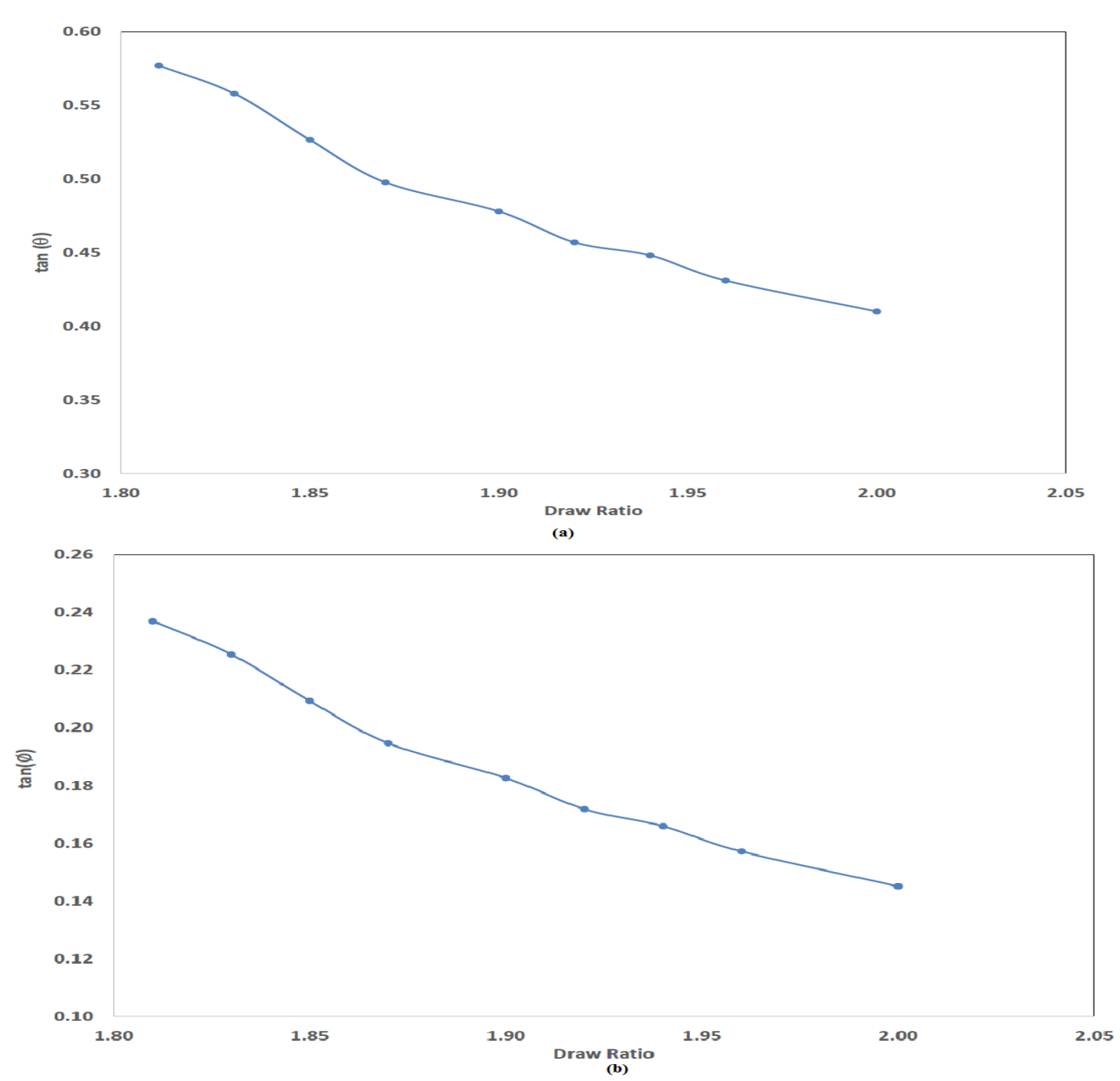

Fig. $7(a, b):$ The variation of the $\tan (\theta), \tan (\emptyset)$ with the draw ratio.

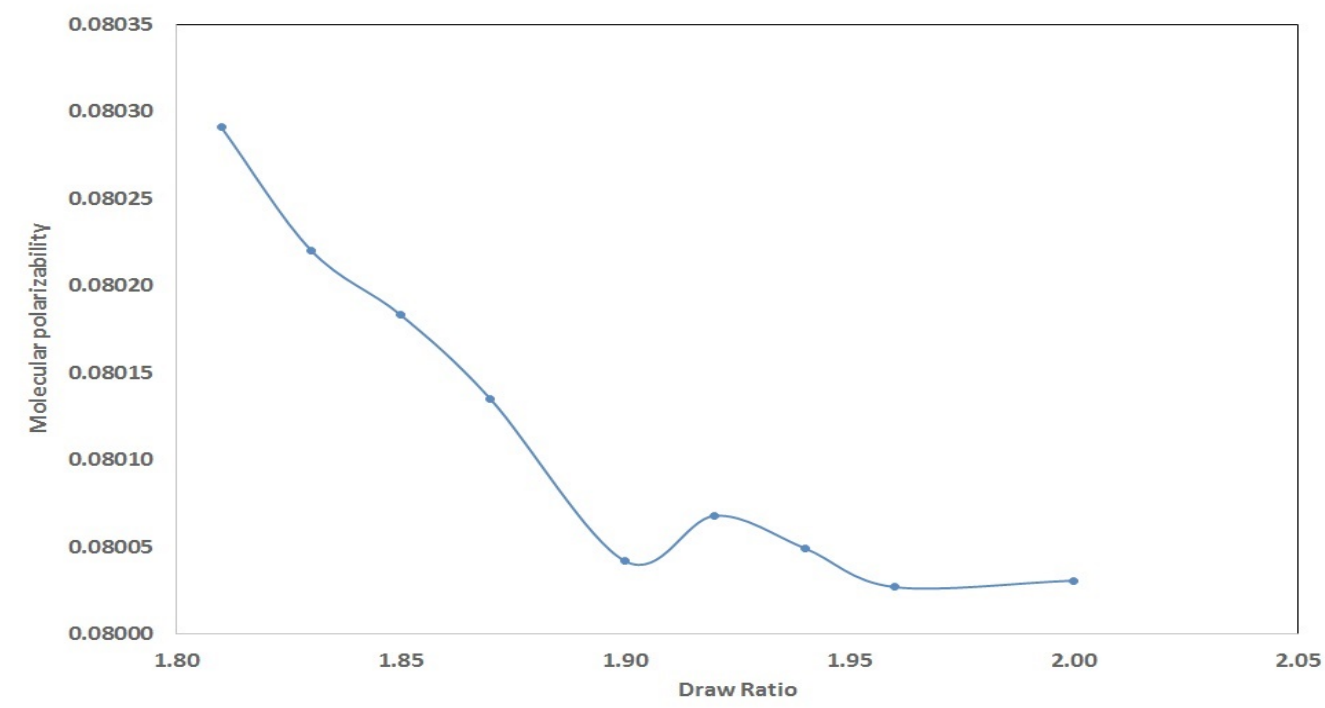

Fig. 8. The molecular polarizability variation with the draw ratio. 


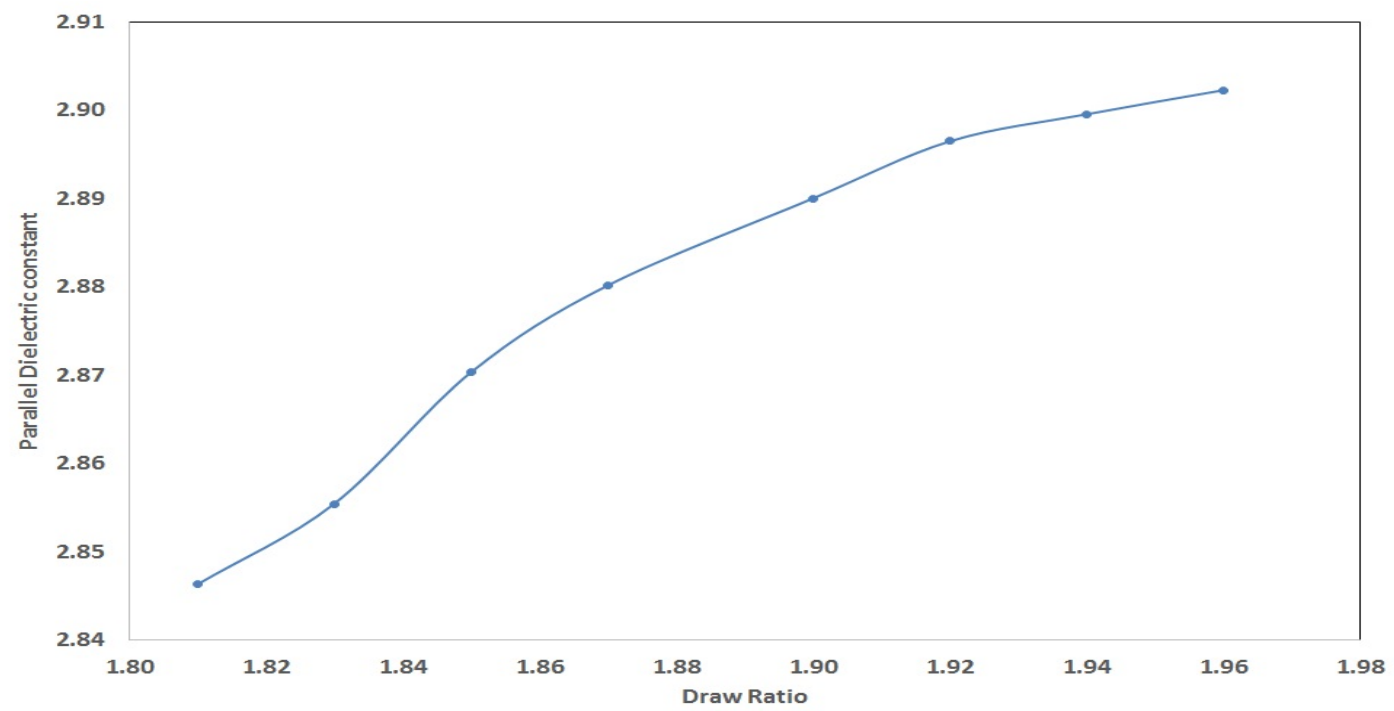

(a)

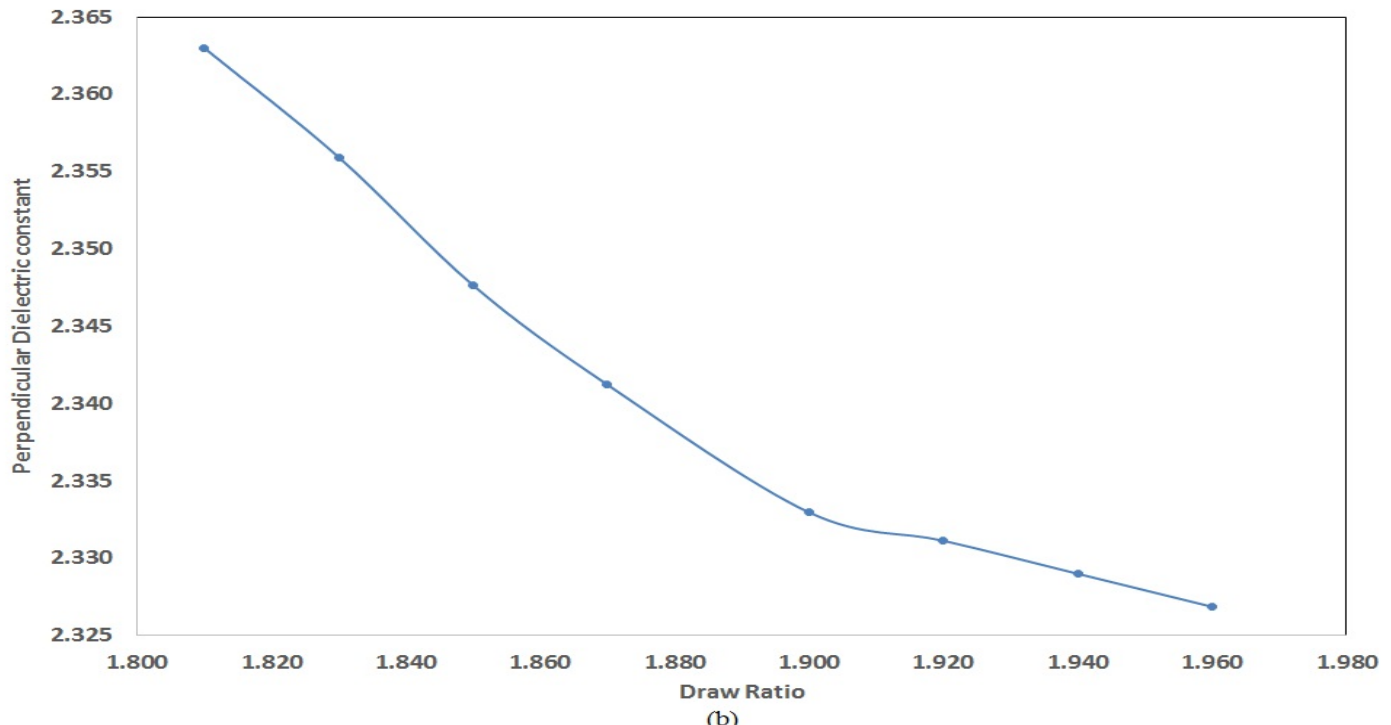

Fig. 9 (a, b): The dielectric constant variation with the draw ratio for parallel and perpendicular direction respectively.

\section{Conclusion}

The cold drawing process was used to calculate the changes in the orientational behavior of the PET fibers. The obtained results show that the drawing process largely influences the different optical, structural, and electrical properties of the PET fibers. With the increased draw ratio, the birefringence and different orientation functions increase. The orientation functions calculated by the optical and mechanical methods demonstrate the appropriate molecular orientation in PET fibers. The different types of orientations show a strong dependence on DR. The interferometric method used presents high accuracy. Hence, this method is recommended for use with the drawing device to characterize different optical and structural properties.

\section{Acknowledgment}

The author would like to thank the Deanship of Scientific Research at Umm Al-Qura University. For the continuous support. This work was supported financially by the Deanship of Scientific Research at Umm Al-Qura University to DR Afaf M Ali. (Grant Code: 17-SCI-1-03-0002). 
TABLE 2. The different structural properties of drawn PET as volume fraction crystallinity and weight fraction crystallinity.

\begin{tabular}{cccc}
\hline Draw ratio & $\mathbf{x}_{\mathbf{v}}$ & $\mathbf{x}_{\mathbf{w}}$ & $\mathbf{1 - \mathbf { x } _ { \mathbf { v } }}$ \\
\hline 1.81 & 0.237112 & 0.259164 & 0.762888 \\
1.83 & 0.227471 & 0.248626 & 0.772529 \\
1.85 & 0.222395 & 0.243078 & 0.777605 \\
1.87 & 0.215794 & 0.235863 & 0.784206 \\
1.9 & 0.203094 & 0.221982 & 0.796906 \\
1.92 & 0.206651 & 0.22587 & 0.793349 \\
1.94 & 0.204111 & 0.223093 & 0.795889 \\
1.96 & 0.201061 & 0.21976 & 0.798939 \\
2 & 0.20157 & 0.220316 & 0.79843 \\
\hline
\end{tabular}

\section{References}

Balcerzyk, E., Wojciech K., Ewa, W and Weronika L., Intrinsic birefringence of nylon 6, Journal of Applied Polymer Science, 26, p. 2573-2580 (1981).

Bilal D., Ali Y., Hüseyin E., Crystallization Behavior of PET Materials. BAÜ Fen Bil. Enst. Dergisi Cilt, 13,p. 26-35(2011).

De Varies A. J., Bonnebat C., Beautemps J., . Journal of Polymer Science: Polymer Symposia,58,p. 109,(1977)

Dumbleton, J. H.. Spin Orientation Measurement in Polyethylene Terephthalate. Textile Research Journal 40,p. 1035-1041,(1970)

Gedde, U. and Jan-Fredrik J., Solidification processes in polymers. Darmstadt; New York: Steinkopff ; Springer (1992)

Hamza, A. A., Sokkar T. Z. N., El-Bakary M. A. and Ali A. M., Interferometric determination of regular and/or irregular transverse sectional shape of homogeneous fibres. Measurement Science \& Technology. 13, P. 1931-1936, (2002)

Huisman, R. and Heuvel H. M. , The effect of spinning speed and drawing temperature on structure and properties of poly(ethylene terephthalate) yarns. Journal of Applied Polymer Science 37, P. 595616 (1989).

Jackson, J. D. , Classical Electrodynamics. New York: J. Wiley (1975).

Keum, J. K., Kim J., Lee S. M., Song H. H., Son Y. K.,
Choi J. I. and Im S. S.. Crystallization and Transient Mesophase Structure in Cold-Drawn PET Fibers. Macromolecules, 36, P. 9873-9878, (2003).

Keum, J.K., Song, H.H., Polymer 26,P. 939. (2005)

Kolb, R., Seifert S., Stribeck N. and Zachmann H. G. Investigation of the high speed spinning process of poly(ethylene terephthalate) by means of synchrotron X-ray diffraction. Polymer, 41, p. 2931-2935, (2000).

Lefebvre, D., Jasse B. and Monnerie L. , Fourier transform infra-red study of uniaxially oriented poly(2,6-dimethyl 1,4-phenylene oxide)-atactic polystyrene blends. Polymer, 22, p. 1616-1620, (1981).

Misra, A. and Stein R. S. Stress-induced crystallization of poly(ethylene terephthalate). Journal of Polymer Science: Polymer Physics Edition, 17, p. 235-257, (1979),

Oultache A. K., Kong X., Pellerin C., Brisson J., Pezolet M. and Prud'homme R. E., Orientation and relaxation of orientation of amorphous poly(ethylene terephthalate). Polymer, 42, P. 90519058 (2001).

Peters R. H., Textile Chemistry, Vol. Amsterdam: Elsevier Scientific (1975).

Pluta M. On the accuracy of microinterferometric measurements of optical-path differences by means of the half-shade method. Journal of Microscopy 93, p. 83-100, (1971).

Purvis, J., Bower D. I. and Ward I. M., Molecular 
orientation in PET studied by polarized Raman scattering, Polymer, 14 p. 398-400, (1973).

Radhakrishnan, J. and Kaito A., Structure formation during the isothermal crystallization of oriented amorphous poly(ethylene terephthalate) films. Polymer, 42, p. 3859-3866, (2001).

Rietsch F, Duckett R A, and Ward I M., Tensile drawing behaviour of PET. Polymer, 20, p. 1133,(1979).

Robertson, G. L.. Food Packing. Principles and practice", NEW York, U Marcel Dekker INC. (1993)

Rossignol, J. M., Seguela R., Rietsch F. and JDupuisLallemand. Intrinsic amorphous birefringence of semi-crystalline polyethylene from combined birefringence and infrared dichroism measurements. Journal of Polymer Science Part C: Polymer Letters, 27, p. 527-532, 1989.

Salem, D. R. ,Orientation and crystallization in poly(ethylene terephthalate) during drawing at high temperatures and strain rates. Polymer Engineering \& Science, 39, p. 2419-2430 (1999).

Sokkar, T. Z. N., El-Bakary M. A. and Ali A. M. , The influence of mechanical cold drawing and drawing velocity on the molecular structure of isotactic polypropylene fiber. Journal of Applied Polymer Science 127, p. 1105-1113 (2013).

Sokkar, T. Z. N., El-Tonsy M. M., El-Bakary M. A., ElMorsy M. A. and Ali A. M. , A novel video optomechanical (VOM) device for studying the effect of stretching speed on the optical and structural properties of fibers. Optics and Laser Technology, 41,P. 310-317(2009).
Sperling, L. H., Introduction to Physical Polymer Science. Hoboken, N.J.: Wiley. (2006).

Stein, R. S., The orientation of polyethylene. POL Journal of Polymer Science, 34,p. 709-720,(1959).

T Yamaguchi, Kim K, Murata T, Koide M, Hitoosa S, Urakawa $\mathrm{H}$ et al, Initial stage of fiber structure development in the continuous drawing of poly(ethylene terephthalate). journal of Polymer Science Part B: Polymer Physics, 46, p. 2126, (2008).

Vander Plaats, G. N., Numerical Optimization Techniques for Engineering

Designs, Colorado Springs, USA, VanderPlaats Research \& Development Inc. (1999).

Venkateswaran, G., Cameron M. R. and Jabarin S. A., Effects of temperature profiles through preform thickness on the properties of reheat-blown PET containers. Advances in Polymer Technology, 17, p. 237-249, (1998).

Williams, David J., Polymer Science and Engineering. Englewood Cliffs: Prentice-Hall , (1971).

Yilmazer, U., Xanthos M., Bayram G. and Tan V. , Viscoelastic Characteristics of Chain Extended/ Branched and Linear Polyethylene Terephthalate Resins. Journal of Applied Polymer Science,75, p. 1371-1377, (2000) 
نسب التبلور و كمية التوجيه في الالياف المشدودة باستخدام تقنيات التداخل الضوئي الثنائي عفاف معوض عبدالمجيا علي

قسم الفيزياء ـ كلية العلوم - جامعة أم القرى ـ المملكة العربية السعودية

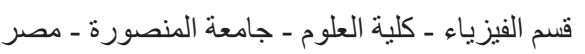

في هذا العمل تم استخدام مقياس التداخل الضوئي الثنائي (بلوتا) لدراسة تاثير عملية الثد علي الخصائئس

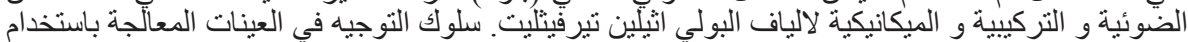

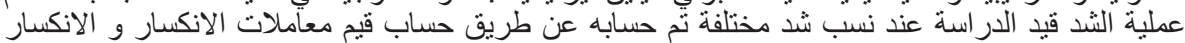

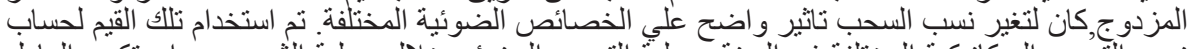

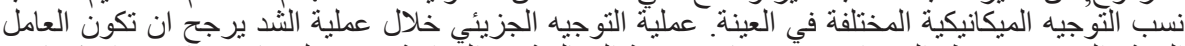

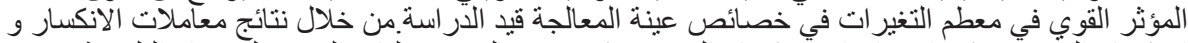

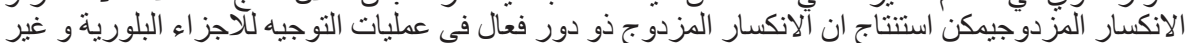

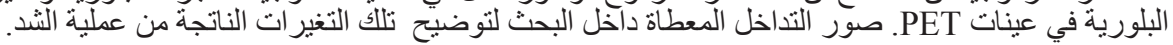

